\title{
La protection des cultures durable : une vraie nécessité mais de nombreux paradoxes
}

\author{
Marc DELOS \\ DRAAF - SRAl, Cité Adminstrative - Bat E, \\ Bd Armand Duportal, 31074 Toulouse cedex \\ <marc.delos@agriculture.gouv.fr>
}

\begin{abstract}
The EU Directive on the Sustainable Use of Pesticides, adopted on 13 January 2009 by the European Parliament encourages the development of an integrated agriculture that would minimize the use of pesticides. The drafters of this text put forward a set of method of management of agricultural pests, including the rotation, the choice of suitable cultivars, different culture techniques or tillage, as well as conditions for driving and to reduce the risks parasites. These are the methods that the following pages are intended to illustrate the examples by focusing as much as possible on oilseed crops. This leads to note however, that some goals may appear paradoxical and, that apart the genetic selection, the rotation appears as the most operational method for which the oilseed crops provide an added value beyond doubt.
\end{abstract}

Key words: pesticide use directive, integrated pest management, rotation, sowing date, tillage, cultivar choice

\section{Introduction}

La satisfaction d'un accès à une alimentation abondante, de qualité et bon marché est certainement le fait majeur du XX ${ }^{\mathrm{e}}$ siècle, même si toutes les régions du monde n'ont pas bénéficié de cette amélioration de façon partagée. Ce progrès dans la « qualité » de vie des hommes en a permis de nombreux autres.

Il est admis que ce développement s'est appuyé sur 3 facteurs techniques principaux :

- I'amélioration variétale ou génétique avec la production de variétés plus performantes, dont les hybrides, tant du point de vue du rendement que de la tolérance aux stress biotiques et abiotiques ;

- I'utilisation d'amendements ou de fertilisants, en particulier d'azote, permettant d'exploiter des sols auparavant dépourvus de toute valeur agronomique puis de faire progresser de façon significative les rendements ; - la mise au point puis l'utilisation, surtout après la seconde guerre mondiale, de produits de protection des plantes (pesticides) destinés à limiter l'impact des bioagresseurs des cultures sur le rendement et la qualité du produit. II convient de noter que les performances de cette technique ont parfois conduit les producteurs de semences à négliger la génétique pour la tolérance aux bioagresseurs correctement contrôlés par les "pesticides", de façon à consacrer l'effort à d'autres facteurs d'amélioration.

À ces trois facteurs techniques, on peut ajouter la maîtrise de l'irrigation, même si cette tech- nique ne s'applique qu'à un nombre limité de cultures, n'étant considérée comme un facteur limitant sous nos climats que pour $20 \%$ des cultures en terres arables, et la mécanisation, mais dans ce cas, l'impact a été plus fort sur la productivité par actif que sur les rendements potentiels eux-mêmes.

Ce rappel contextuel et son illustration sont apparus indispensables pour aborder la thématique qui est l'objet de notre communication dans la mesure où nous ne retenons depuis 5 à 10 ans que les effets négatifs de ces techniques avec des effets à long terme sur la santé des applicateurs, la contamination des eaux et des milieux par les fertilisants et les produits de protection des plantes. Ces risques et contaminations ont depuis conduit à de nombreuses restrictions et encadrements d'emploi ou à la mise en œuvre de dispositifs de protection des applicateurs ou de l'environnement avec notamment la généralisation de zones tampons, dont les bandes enherbées destinées à bloquer dans la parcelle tout ou partie des pesticides et des fertilisants apportés parfois encore en excès dans les parcelles.

C'est dans cette perspective d'amélioration, au-delà des efforts déjà accomplis qui ne doivent pas être sous-estimés, que ces quelques pages ont été rédigées afin d'illustrer et de commenter les propositions contenues dans la future directive européenne sur l'utilisation durable des pesticides pour la mise en œuvre de différents outils de lutte intégrée.

\section{Les principes de lutte intégrée développés par la directive sur I'utilisation durable des pesticides}

Le 13 janvier 2009, la directive du Parlement européen et du Conseil instaurant un cadre d'action communautaire pour parvenir à une utilisation des pesticides compatible avec le développement durable était adoptée après plusieurs mois de travaux.

Si son objectif est de stimuler l'adoption de plans nationaux d'action afin de « réduire les risques et conséquences de l'utilisation des pesticides » pour la santé humaine et l'environnement, la directive fait référence au développement de mesures destinées à promouvoir une gestion intégrée de la lutte contre les parasites de plantes et des méthodes de contrôle alternatives aux pesticides. Elle rappelle en parallèle la nécessité d'organiser la surveillance des bioagresseurs et I'utilisation de tout moyen de raisonnement permettant I'utilisation la plus réduite possible des moyens de lutte chimique en les optimisant (réponse aux questions: Où traiter? Quand traiter ?).

Différents moyens ont été relevés par la directive comme pertinents pour éviter ou réduire I'utilisation de produits phytopharmaceutiques, nous noterons l'exhaustivité des rédacteurs de ce document réglementaire, lorsqu'ils 
mentionnent parmi les méthodes de prévention et/ou l'éradication des organismes nuisibles :

- la rotation de cultures ;

- I'utilisation de techniques de culture appropriées (faux semis, choix de dates et densités des semis, les semis sous couvert, la « pratique aratoire conservative ", et le semis direct) ;

- I'utilisation, lorsque c'est approprié, de cultivars résistants/tolérants et de semences et plants normalisés/certifiés ;

- I'utilisation équilibrée de pratiques de fertilisation, de chaulage et d'irrigation/de drainage ;

- la prévention de la propagation des organismes nuisibles par des mesures d'hygiène (nettoyage régulier des machines, équipement, silos);

- la protection et le renforcement des organismes utiles importants (protection des plantes appropriées ou l'utilisation d'infrastructures écologiques à l'intérieur et à l'extérieur des sites de production).

Cet inventaire est complété par la référence aux méthodes biologiques, physiques et autres méthodes non chimiques durables, en prenant soin de préciser «si elles permettent un contrôle satisfaisant des bioagresseurs » qui traduit un bon réalisme des rédacteurs du projet de directive.

Nous nous proposons donc de reprendre chacune des méthodes alternatives listées dans la directive et de les contextualiser avec des exemples pris essentiellement dans les différentes cultures oléagineuses, pour en confirmer le bien-fondé. Cependant, le recours aux méthodes agronomiques n'étant pas univoque dans les domaines du vivant qui caractérisent l'agriculture, les limites et souvent les paradoxes liés à la mise en œuvre des différentes méthodes seront soulignés au fur et à mesure du développement.

\section{La rotation des cultures : base de l'agriculture puis de I'agronomie}

La rotation des cultures constitue le premier moyen de lutte prophylactique auquel il est généralement fait référence. Elle est efficace sur les nombreux parasites des cultures pour lesquels la source de contamination initiale a une origine essentiellement endogène à la parcelle (sclérotina des dicotylédones, mildiou du tournesol et du colza, phoma du tournesol pour partie...). L'agriculteur, avec les outils de gestion moderne des bioagresseurs, est passé à des rotations simplifiées $n$ 'incluant que 2 cultures dans de nombreuses régions françaises, avec de nombreuses variations allant de 6 cultures judicieusement choisies à la monoculture qui caractérise surtout la production céréalière (blé et surtout maïs). De fait, il est exceptionnel que deux cultures oléagineuses se succèdent, il s'agit d'une opportunité en vue de l'agriculture durable.

Les rotations doivent se raisonner en fonction des possibilités de multiplication de l'inoculum sur les cultures précédentes. Les pathogènes polyphages se développant sur plusieurs cultures génèrent des contraintes supplémentaires dans le choix des successions culturales ou des techniques de travail du sol. C'est, par exemple, le cas des plantes sensibles au sclérotinia.

Ce phénomène n'existe pas dans le cas de successions céréales/dicotylédones dont les effets sont dans la plupart des cas positifs en matière de risques parasitaires. En effet, même si la survie de l'inoculum, sous forme de sclérotes par exemple, dépasse largement la durée de la rotation, l'absence de cultures hôtes permet de réduire le stock d'inoculums du sol, la pratique systématique du labour, ou l'absence de cette pratique peut compliquer cependant cette assertion.

La rotation peut être particulièrement efficace pour lutter contre les mauvaises herbes, notamment dans le cadre de successions de cultures implantées à des périodes différentes (cultures d'hiver, de printemps et d'été), principalement en permettant des interventions culturales assurant la destruction de la flore adventice issue de la culture précédente avant la production de semences. Cette alternance joue également par la rupture de l'adaptation d'une flore au cycle d'une culture, adaptation favorisée par la monoculture ou une rotation simplifiée. Indépendamment de cet effet direct, la rotation de cultures de dicotylédones avec des cultures de graminées ouvre des possibilités de désherbage plus raisonné et favorise une lutte contre l'émergence d'adventices résistantes.

Quel que soit le système de culture, la rotation des cultures est quasi systématiquement une pratique de gestion optimale des parasites des plantes. En l'absence de travail du sol, elle peut prendre encore plus d'importance, surtout pour interrompre le cycle de vie des insectes, le cycle des maladies et celui des mauvaises herbes. Les ravageurs les plus sensibles à la rotation sont ceux pour lesquels le spectre d'hôte est limité et dont le développement a lieu dans le sol. C'est notamment le cas des dégâts de taupins qui sont fortement influencés par les précédents de cultures présentant un couvert végétal pendant plus de deux ans, telles les cultures fourragères et les prairies, plus favorables à l'activité de ponte des adultes mais aussi empêchant le travail du sol, moyen de destruction des pontes de l'insecte s'il est réalisé en conditions séchantes.

Au bilan, la rotation apparaît comme un outil de régulation majeur des bioagresseurs inféodés à la parcelle dont les maladies portées par le sol telles le mildiou du tournesol, même si des cycles aériens sont possibles. Les effets de la rotation sont intermédiaires pour le sclérotinia et les fusarioses dont les ascospores sont peu mobiles. Ils sont fonction de la surface de la parcelle. L'efficacité est faible pour les parasites mobiles avec échange entre parcelles voisines. Cette absence d'effet est d'autant plus notable que la mobilité de ces derniers est grande et que la surface des parcelles est réduite. La rotation ne joue plus sur les organismes à forte mobilité comme les lépidoptères et les champignons tels que les oïdiums et les rouilles. L'oidium du colza étant en France le représentant le plus nuisible de ce groupe de bioagresseurs mobiles et la rouille du soja, non introduite en Europe, au plan international.

La principale contrainte qui s'oppose à la rotation est d'ordre économique, lorsqu'une culture présente un bilan économique bien supérieur à celles qui peuvent lui succéder dans le cadre de la rotation, y compris en prenant en compte l'impact des bioagresseurs ou les risques agronomiques et les surcoûts qu'ils entraînent tout au moins à court terme. Cela a pu exister pour le tournesol au cours des années 1980-1990 mais reste exceptionnel pour les oléagineux, quasi systématiquement en rotation avec des céréales.

\section{Le labour et le travail du sol : moyen de lutte polyvalent mais perturbateur de l'écologie du sol}

La pratique du labour par I'utilisation de la charrue pour travailler le sol et retourner la terre est ancienne en Europe. Elle se répand dès le $\mathrm{XI}^{\mathrm{e}}$ siècle sur les terres «lourdes » du nord de la France avec le développement des charrues à soc primitives. L'efficacité de la charrue est alors surtout mesurée en termes de facilitation de préparation du sol avant le semis, en particulier dans les sols riches en argile exposés à la réduction des mottes par l'action du gel. Après une phase de fort développement de la traction animale au cours du XIX ${ }^{\mathrm{e}}$ siècle, les derniers « progrès » significatifs, entre 1920 et 1950, sont liés à la traction mécanique de la charrue suite au développement du tracteur, qui ne se généralisera en France qu'après 1950. Ce développement a facilité la mise en œuvre du labour mais a aussi modifié la pratique qui ne peut plus être désormais perpendiculaire à la pente et favorisera l'érosion des sols dans les terrains en pente. Si le labour est attaché à la tradition agricole européenne, les techniques sans labour, importées d'outre-atlantique, sont plus favorables à la durabilité des sols et à leur écologie. Sous nos climats, ces techniques simplifiées favorisent nettement les limaces, en raison de l'absence 
de perturbation en période de sécheresse, mais aussi au travers de la litière végétale laissée à la surface du sol, parfois les mouches. Cependant, c'est le roulage du sol qui constitue le principal moyen de réduction des limaces. Pour les taupins, les avis divergent avec un constat de populations réduites lorsque le travail du sol est réalisé en conditions sèches exposant les œufs et les jeunes larves de l'insecte, cet effet est actuellement difficilement dissociable de celui du couvert végétal permanent caractérisant les parcelles dans lesquelles le travail du sol a été abandonné, couvert qui favorise fortement les pontes de l'insecte. L'effet du labour est également notable sur d'autres insectes vivant dans ou au niveau du sol, tels les hannetons et les scutigerelles pour lesquels le travail du sol en général et le labour en particulier participent au contrôle au même titre que la rotation. S'agissant des hannetons, ce sont les outils de travail du sol animés utilisés lorsque les larves sont à la surface du sol, fin printemps-été, qui donnent les meilleurs résultats en termes de destruction.

L'absence de travail du sol peut se manifester de façon très indirecte sur les ravageurs, en lien avec le maintien des relevées de la culture précédente lorsque cette dernière constitue le support trophique indispensable à l'espèce considérée. Le cas de la chrysomèle du maïs (Diabrotica virgifera virgifera) est emblématique de ce phénomène avec une prise en défaut de la politique d'éradication par la rotation de la culture du maïs, suite à des relevées de maïs dans les cultures suivantes en particulier le soja. L'absence de labour après la récolte du maïs permettant à des fractions d'épis tombées au sol lors de la récolte de produire de nouvelles plantes de maïs dont les racines sont indispensables pour le développement des larves de l'insecte. Ce phénomène a été observé dans du soja succédant à du maïs dans des zones de lutte obligatoire en Alsace en 2008, gestion compliquée par l'interdiction de la trifluraline sur la culture. Ces plants de maïs indésirables seront à l'origine du maintien de l'insecte dans la parcelle et seront de nature à contrarier l'effort entrepris dans le cadre d'une politique d'éradication.

Enfin, parmi les ravageurs sur lesquels l'effet du travail du sol est déterminant, nous citerons les vertébrés terrestres (par exemple : les campagnols) dont les dégâts et populations sont directement liés à la surface de prairies et de bordures en herbe non travaillées. Le caractère cyclique des pullulations et l'interaction avec d'autres facteurs de régulation, avec un pas de temps d'une dizaine d'années entre deux pullulations, ne permet pas d'évaluer correctement à la fois toutes les conséquences de la réduction du labour depuis 2001 et de l'implantation des bandes enherbées, devenue obligatoire le long des cours d'eau sur l'évolution des populations de ces organismes.

Sur les adventices, le travail du sol via le labour contribue à l'enfouissement des semences d'adventices produites au cours de la campagne précédente. La meilleure efficacité d'enfouissement est obtenue avec le labour, plus efficace que les travaux profonds sans retournement, eux-mêmes plus efficaces que les travaux superficiels. La majorité des levées est issue de semences germant dans les horizons les plus superficiels, l'enfouissement en profondeur contribue à limiter les infestations dans la culture suivante. Cependant, le travail du sol a aussi pour effet de remonter des semences anciennes depuis les horizons profonds.

Pour certaines espèces aux semences fragiles (vulpin, bromes, ray-grass, gaillet...), le séjour en profondeur pendant un an ou plus induit des pertes de viabilité importantes, de sorte que le labour enfouit plus de semences viables qu'il n'en remonte en surface. En revanche, les espèces à stock semencier persistant sont plutôt favorisées en labour et défavorisées en nonlabour.

Même pour les espèces sensibles à l'enfouissement, le labour systématique tous les ans n'est pas forcément la solution la plus efficace.

Si les dates de semis sont très contrastées sur la rotation (par exemple : la succession maïs-blé), les espèces infestant les différentes cultures de la rotation ne sont pas les mêmes. Le fait de ne labourer qu'un an sur deux permet d'augmenter la durée de séjour des graines dans le stock semencier, et donc d'augmenter le taux de mortalité naturelle des semences dans le sol. La mise en place du non-labour doit donc intégrer des choix de cultures et de rotations pour éviter le développement favorisé de certaines mauvaises herbes (bromes, vulpin, vulpie, raygrass, agrostis, gaillet, géranium, et pour les cultures d'été, panic, sétaires et digitaires...), avec des dates de semis contrastées.

De façon très schématique, le labour réduit les populations de graminées alors que le semis direct est globalement plus favorable au contrôle des dicotylédones annuelles [1].

La litière végétale non enfouie oblige à repenser le désherbage en utilisant davantage d'herbicides et en préférant les produits foliaires car elles limitent I'efficacité des herbicides racinaires (pendiméthaline, trifluraline, chloroacétamides, pour partie urées substituées), le poids des herbicides foliaires dans la gestion des adventices (fops, dimes, sulfonylurées, glyphosate...) est augmenté, fragilisant d'autant ces solutions par la pression de sélection sur des population résistantes d'adventices. Cet effet s'ajoute à celui de l'absence d'action herbicide immédiate ou différée permise par le labour.
Pour compenser l'abandon du labour, il est fait appel au travail du sol superficiel pendant l'interculture qui a deux fonctions :

- d'une part, il détruit les mauvaises herbes présentes après la récolte qui ne subissent plus la concurrence de la culture et peuvent donc produire un grand nombre de semences. Cette première fonction exige une intervention avant que les graines soient viables. La destruction des espèces présentes par des moyens mécaniques peut remplacer un désherbage chimique s'il est réalisé par temps sec [2] ;

- d'autre part, il stimule les levées pendant l'interculture de façon plus ou moins importante selon les conditions climatiques, réduisant d'autant le potentiel d'infestation dans la culture suivante (à condition de détruire efficacement les plantules levées avant le semis).

Le faux semis, puisqu'il s'agit de cette technique, pour être efficace suppose un intervalle suffisamment long entre la récolte de la culture précédente et le semis de celle qui suit, intervalle difficilement réalisable entre une céréale à pailles et un colza. II suppose également des conditions climatiques permettant la levée des adventices donc des pluies suffisantes pour permettre une humectation du sol. Enfin, un seul faux semis est rarement suffisant, suivant le stock de semences d'adventices à détruire, deux ou trois opérations successives seront nécessaires pour obtenir une réduction suffisante du stock semencier.

De façon pratique, deux stratégies sont possibles pour les cultures sans labour selon L. Bonin (Arvalis) : la mise en œuvre de faux semis telle que développée précédemment, ou bien assurer une perturbation minimale du sol évitant la levée de nouvelles adventices.

Sur le plan du transfert des herbicides dans les milieux, si les TSL sont favorables pour réduire le transfert des herbicides en sols battants, dans les sols argileux, en l'absence de travail du sol avant la réhumectation de ce dernier, les risques de transfert sont élevés à l'automne du fait d'un ruissellement généralement plus important.

Pour les maladies, les techniques de travail du sol en tant que moyens de lutte sont indissociables de la rotation dans leur prise en compte. Il convient en outre de distinguer leur effet sur les champignons en fonction de la mobilité de leur inoculum et de leur capacité à se développer ou à se maintenir sur des résidus de culture. Nous distinguerons les maladies à inoculum partagé (IPA), ascospores (mutualisation de la contamination entre parcelles voisines), des maladies à inoculum propre à la parcelle (IPR), conidies ou champignon du sol.

L'enfouissement des résidus de culture constitue un moyen efficace dans le cas de parasites pour lesquels la contamination est liée à l'émission de spores, ascospores ou conidies. 
Le phénomène est régulièrement observé dans le cas du phoma du colza : la quantité d'ascospores de Leptosphaeria maculans piégée par les capteurs de spores est réduite dès l'instant où les résidus de culture infectés sont enfouis pour la préparation des semis des céréales d'hiver. Un effet significatif est généralement observé avec les maladies à sclérotes telles que Sclerotinia sclerotiorum mais avec des performances qui varient en fonction de la vitesse de dégradation du sclérote dans le sol.

Outre les effets d'enfouissement bien identifiés, le labour réduit I'humidité et la teneur en matière organique du sol et en abaisse la température et le $\mathrm{pH}$. Ces changements du microenvironnement influent sur les populations de micro-organismes du sol.

II existe une analyse divergente entre les partisans du non-labour, moyen de lutte contre l'érosion, et ceux qui soulignent les effets pervers de l'abandon de l'enfouissement des résidus de culture en interaction avec la nature du précédent. Dans tous les cas, une évaluation correcte du phénomène de mutualisation de l'inoculum, liée à la proximité de parcelles sources d'ascospores dans lesquelles les mesures prophylactiques de base n'ont pas été mises en œuvre, sera de nature à trancher le débat, même si de nombreux éléments permettent déjà d'asseoir le principe de l'effet positif global du labour pour lutter contre les maladies.

Le terme de « techniques aratoires conservatives » est choisi à dessein dans la directive afin de souligner l'effet du labour sur les phénomènes d'érosion du sol, en particulier dans les parcelles en pente. En parallèle avec l'impact sur l'érosion des sols, de nombreuses espèces notamment de la microfaune, neutres ou utiles, vivant dans le sol, sont perturbuées par la pratique du labour : parmi les espèces les plus sensibles à cette pratique, nous listons les vers de terre [3] et les araignées. Le passage d'un travail du sol avec labour à un système de semis sur travail superficiel ou de semis direct augmente notablement le volume des galeries de vers de terre ainsi que celui des rejets de vers de terre en surface dans des successions de cultures annuelles sous climat continental sur des sols de limons profonds. La moindre perturbation des populations de vers de terre et l'augmentation de la masse organique présente à la surface du sol liée à l'abandon du labour, modifient la structure de ce dernier. La réduction du travail du sol se traduit en quelques années par une amélioration de la stabilité de la structure [46]. Les changements de stabilité de la structure peuvent être détectés dès 2 à 3 ans après le changement de pratique culturale.

Au bilan, le travail du sol notamment via le labour apparaît comme la méthode de lutte idéale contre les parasites liés aux résidus de cultures ou qui se maintiennent à la surface du sol. Elle présente une efficacité faible pour les champignons biotrophes. L'effet est d'autant plus notable que la mobilité des organismes cibles est faible ou que cette lutte est conduite à l'échelle territoriale, mais l'effet de la technique sur les phénomènes d'érosion, son impact sur la biodiversité du sol et pour l'agriculteur, le coût du fuel et le temps de travail liés à sa mise en œuvre tendent à réduire son emploi. L'absence de labour peut être partiellement compensée par un broyage des résidus et une incorporation superficielle, méthode recommandée pour le phomopsis du tournesol et le phoma du colza même si l'efficacité reste inférieure à celle de l'enfouissement .

\section{L'exportation pour valorisation énergétique ou industrielle : I'avenir de la gestion des résidus de récolte}

À défaut de labour, la valorisation énergétique des résidus de culture permettrait de gérer différents bioagresseurs non telluriques, la référence aux techniques de gestion des résidus est récurrente.

Ce moyen de gestion constituerait en outre une avancée considérable pour lutter contre des maladies comme le phomoposis du tournesol, divers phoma, divers insectes se conservant dans les résidus de culture. Seuls les bioagresseurs telluriques et les champignons essentiellement biotrophes échapperont à cette évolution et maintiendront une pression au moins équivalente sur les cultures. Cette exportation ne pourra cependant concerner qu'une partie des résidus pour assurer la restitution de matière organique aux sols.

\section{La suppression des relevées de la culture et adventices hôtes : pour assurer une pleine efficacité à la rotation et l'étendre hors de la parcelle}

Pour être efficace, la rotation des cultures suppose, outre l'absence de flux d'inoculum de maladie ou d'insectes depuis les parcelles voisines, l'absence de maintien de l'insecte ou de la maladie dans la culture non hôte, via la présence de «repousses » de la culture précédente.

Des exemples illustrent le caractère indispensable de ce contrôle, telle la multiplication de Diaporthe helianthisur des repousses de tournesol dans des cultures de soja irriguées, conditions particulièrement favorables pour le déve- loppement de la maladie une année sèche ou le maintien de Plasmopara halstedii sur les repousses de tournesol dans les céréales à pailles qui suivent le tournesol, annulent les bénéfices de la rotation.

Dans le cas du colza, les repousses laissées après la culture pendant une partie de l'automne présentent l'avantage de fixer l'azote mais peuvent aussi servir de refuge pour divers insectes de la culture et favorisent la multiplication des limaces. Elles empêchent enfin la pratique du labour, moyen efficace de réduire l'inoculum en cas d'un précédent fortement contaminé par le phoma.

C'est également le cas d'adventices appartenant à la famille des crucifères vis-à-vis de la hernie du choux (Plasmodiophora brassicae) ou de diverses adventices composées vis-à-vis du mildiou du tournesol qui maintiennent les bioagresseurs dans des cultures non hôtes telles les céréales à pailles. Cette nuisibilité indirecte liée à la présence d'adventices dans la culture rend les seuils de nuisibilité de ces dernières peu adaptés. Le seuil de tolérance devenant dans le cas d'un rôle d'hôte relais difficile à établir et dans tous les cas très en deçà de la nuisibilité directe. Si ce rôle est identifié, la lutte prophylactique passe par l'élimination de ces plantes de la culture non hôte.

\section{Impact des dates de semis}

Jouer sur les dates de semis, c'est utiliser un effet antagoniste à celui du climat en jouant sur deux mécanismes différents, soit en réduisant le nombre de cycles possibles, par une diminution de la durée végétative, soit en déplaçant la phase sensible de la plante par rapport à une phase climatique favorable aux bioagresseurs. Dates de semis et précocités des variétés permettent d'obtenir ces effets.

\section{Effet sur le nombre de cycles des maladies ou des I'insectes}

Le phénomène épidémique est permis par l'effet du climat sur la multiplication des champignons pathogènes ou des insectes ravageurs dans un système de culture donné. Plus la durée de végétation sera importante, plus les organismes nuisibles seront en mesure d'accomplir un nombre de cycles important. C'est une des raisons pour lesquelles avant la mise en œuvre de la protection fongicide systématique, les semis des céréales étaient réalisés sur la fin de l'automne pour limiter, entre autres, les conséquences du parasitisme sur le rendement.

Si de nombreux exemples sont observés sur céréales, les observations sur oléagineux sont moins fréquentes, des maladies comme le sclérotinia du colza, le phomopis du tournesol 
étant, de fait, monocycliques. Sur colza, les dégâts de phoma correspondent aux premiers cycles du champignons. Seuls l'alternaria, la cylindrosporiose et le pseudocercosporella du colza permettraient l'observation de ce phénomène. Sur soja, à l'étranger le phénomène est mieux renseigné, le semis précoce est responsable d'une plus grande attaque de la culture pour les maladies causées par Alternaria alternata, Colletotrichum dematium, Cercospora kikuchii, C. truncatum.

\section{Décalage de la phase de réceptivité maximale par un semis décalé}

S'agissant des oléagineux, c'est la tactique dite $d^{\prime}$ « esquive » qui se révèle la plus efficace sur les maladies. C'est le cas du mildiou du tournesol dû à Plasmopara halstedii où un simple décalage d'une décade de la date de semis peut permettre d'éviter une phase climatique pluvieuse favorable aux contaminations primaires par le champignon avant la levée, stade où la plante est le plus sensible, et ainsi réduire de façon considérable l'épidémie [7].

Une date de semis précoce permet de limiter les conséquences des contaminations automnales de phoma sur les cultures de colza [8], les stades sensibles étant intervenus précocement avant la maturité des ascospores.

L'effet des semis tardifs permettant d'éviter le recours aux fongicides est également observé avec le phomopsis du tournesol dû à Diaporthe helianthi. Dans ce cas, le décalage de la date de semis ne permet pas spécifiquement d'éviter ou de réduire la phase sensible aux contaminations mais de décaler cette dernière dans le temps de façon à l'observer fin juin à mijuillet, avec une forte probabilité d'observer de façon concomitante des températures élevées propres à bloquer ou détruire le champignon sous forme de mycélium à l'intérieur des feuilles. Dans le cas des semis précoces, le champignon a pu atteindre la tige et n'est plus sensible à l'effet du climat.

Pour une culture de soja, le semis retardé défavorise Diaporthe phaseolorum [9].

Une levée rapide et régulière de la culture favorise le contrôle ultérieur des adventices, ce qui renvoie au choix de la date optimale de semis en fonction de la température et de l'humidité du sol. Un semis trop précoce au printemps (tournesol) peut entraîner un développement $d^{\prime}$ adventices peu exigeantes en température. À l'inverse, un semis tardif de céréales d'hiver permet de bien contrôler la flore automnale et de repousser la vague de levée principale des adventices vers le printemps, lorsque le couvert cultivé est bien installé. Le semis différé ou retardé minimise aussi la compétition envers les cultures qui s'implantent et réduit I'utilisation d'herbicides ou de techniques culturales de désherbage (herse étrille...).

La mise en place de cultures de printemps, ou de cultures au semis encore plus tardif, si le système de production le permet (soja, maïs), présente un double avantage : elle permet d'une part de diminuer la densité de vulpins au semis, $d^{\prime}$ autre part d'ouvrir la gamme de familles herbicides utilisables pour contrôler les plantes résistantes.

Pour les insectes, le maïs ou le tournesol, au stade plantule, il s'établit une "course de vitesse » entre l'action des déprédateurs souterrains et la croissance de la jeune plante. De ce fait, les semis précoces, dont la croissance est souvent plus lente en raison de températures moyennes généralement inférieures ou de minima plus bas sont ceux qui en moyenne subissent les plus fortes attaques.

La croissance plus lente des plantes augmente leur période de sensibilité à des déprédateurs comme les taupins (Agriotes sp.), les scutigerelles (Scutigerella immaculata et Scutigerella sp), les nématodes (Ditylenchus dipsaci, Pratylenchus sp.) ou encore les mouches des semis (Delia platura, etc.). Attendre quelques jours pour semer en conditions plus favorables, afin d'obtenir une croissance plus rapide, permet souvent de moins exposer la culture.

\section{Choix variétaux et lutte génétique}

Les choix variétaux jouent sur les parasites des plantes via la génétique, il peut s'agir d'effets directs en essayant de distinguer les situations de résistance vraie avec les conséquences fréquentes de cette résistance en termes de fragilité lorsque la résistance est d'origine monogénique, de type "gène pour gène ", résistance souvent qualitative, polygéniques ou indirects liés notamment à une morphologie particulière d'un organe, limitant la contamination ou l'attaque par le ravageur.

La mise en œuvre de la génétique la moins sensible constitue un des moyens les plus opérationnels pour éviter le recours à la lutte chimique. II convient cependant d'identifier les moyens génétiques reposant sur une résistance de type vertical (résistance qualitative) correspondant à la présence, dans la variété, d'un ou plusieurs gène(s) évitant la contamination par les pathotypes les plus représentés. Ce type de résistance, souvent de haut niveau, est assez rapidement remis en question dès lors que la variété ou les variétés portant des gènes de résistance identiques occupent une part importante de l'espace dans une région donnée. Ce phénomène est régulièrement observé avec le mildiou du tournesol dû à Plasmopara halstedii au cours des années 1980-1990 (races 703 et 710) puis à nouveau au cours des années 2000 (races 304...), il en est de même pour le colza dans le cadre des gènes de résistance à Leptosphaeria maculans utilisés (cas du contournement de la résistance à RLM1 dans les années 1990) ou sur tournesol pour la résistance des cultivars à l'orobanche cumana, principale plante parasite de la culture avec des contournement fréquents.

Dans le cadre de la lutte contre Leptosphaeria maculans, phoma du colza, les gènes $R \operatorname{lm} 1$ et RIm4, fréquemment utilisés par les sélectionneurs dans les variétés à bon comportement en 2005 sont déjà plus ou moins contournés dans de nombreuses zones de culture en France. En 2007, quelques variétés récentes possédaient un nouveau gène très efficace (RIm7) mais ce dernier va subir un contournement dès lors que son exposition aux populations du pathogène sera significative.

De nombreux exemples de possibilités s'appuyant sur le choix de variétés tolérantes (résistance quantitative) ou à sensibilité réduite existent, citons en vrac les variétés de tournesol développées à compter de la fin des années 1990 vis-à-vis de Diaporthe helianthi.

Ce moyen agronomique est très spécifique de l'organisme cible voire de la race de l'organisme cible; cela suppose l'accumulation de gènes permettant de contrôler les différentes races de l'organisme nuisible et des différents organismes nuisibles avec les limites de réalisation de ce type de construction.

Pour des inoculums peu mobiles, il est aussi possible d'envisager l'utilisation en alternance des variétés dont la résistance s'appuie sur des gènes différents afin de limiter, ralentir ou éviter la sélection de populations ou races contournant les génétiques variétales développées [10]. Dans le cas extrême qui suppose une parfaite organisation de la profession, il est possible $d^{\prime}$ envisager de gérer ces phénomènes par une répartition en mosaïque au niveau de l'espace. Ce cas d'école reste pour l'instant une construction intellectuelle. En revanche, la diversité des sources de résistance sur le terrain, même non organisée, permet de réduire les risques épidémiques et assure une gestion plus durable des résistances variétales (cas de la gestion de la rouille jaune du blé en Suisse).

La lutte génétique reposant sur des variétés tolérantes au pathogène s'inscrit davantage dans une agriculture durable. C'est le cas pour le phomopsis du tournesol, maladie pour laquelle les progrès génétiques entre 1993 et 1998 ont permis de faire reculer le recours à la lutte chimique. L'efficacité n'étant pas totale, la mise en œuvre d'autres moyens prophylactiques peut s'avérer nécessaire dans les situations les plus exposées (fonds de vallée, zones affectées l'année précédente). 


\section{Couvert végétal réduit et fertilisation : densité et azote}

L'augmentation des densités de semis, voire la réduction des écartements entre rangs, permet d'économiser des herbicides par la couverture précoce du sol qui augmente la mortalité des plantules d'adventices, réduit la croissance et limite la production semencière. Mais, cette forte densité de peuplement peut favoriser la propagation de certaines maladies cryptogamiques chez le blé (oidium, septorioses, piétin verse) et le tournesol (proximité des pieds, maintien de l'humidité sous couvert) et augmente les risques de verse. Pour le soja, l'infection par Diaporthe phaseolorum est accentuée par une augmentation de la densité des plantes ainsi que par un taux d'azote élevé. Par ailleurs, en conditions sèches, il peut en résulter une consommation trop rapide de la réserve en eau du sol. Enfin, s'agissant notamment de semences d'hybrides, le coût supplémentaire des semences peut être excessif par rapport au résultat. Le choix d'une variété moins couvrante ou d'une plus faible densité de semis pourra s'accompagner d'un écartement plus grand permettant le binage du tournesol. Si le type de sol ou le climat ne permet pas de garantir une lutte mécanique satisfaisante, il est généralement recommandé pour exploiter cet effet d'opter pour des écartements réduits, en choisissant plutôt des variétés tolérantes aux maladies.

En complément de la rotation et incluse dans le programme de contrôle alternatif, la rotation peut faciliter l'utilisation de cultures dites " étouffantes » telles que la luzerne qui permet d'améliorer l'efficacité du système. Ce choix peut supposer des rotations longues et une valorisation des produits issus de la culture dite « étouffante » mais améliore aussi sensiblement le bilan énergétique du système en raison de la restitution de l'azote au final dans la parcelle. L'évaluation du système sur certains insectes du sol qui peuvent être favorisés par l'introduction de couverts végétaux permanents sans labour tels les taupins et les hannetons doit cependant être actualisée.

L'effet de la réduction des apports azotés est particulièrement manifeste sur différents oïdiums (colza). Cet effet est direct sur la sensibilité de la plante ou indirect sur le microclimat via l'augmentation du volume du couvert végétal. Cet effet de la fertilisation azotée est observé dès lors qu'une phase favorable à l'oïdium est concomitante à l'apport d'azote ou lui succède. L'effet de la fertilisation est peu ou pas sensible sous climat peu favorable à I'oïdium (climat pluvieux) ou dès lors que malgré des conditions à faible précipitation qui rendent le développement de l'oïdium possible ne permettent pas l'absorption par la plante de l'azote apporté et empêche son effet. Le lien entre fertilisation azotée et développement des maladies, oïdium des céréales et du colza en particulier, doit tenir compte de l'utilisation de cet apport avant la phase climatique favorable à l'oïdium et non pas de la quantité épandue sensu stricto. Les années caractérisées par une sécheresse prolongée sont révélatrices du phénomène.

Des travaux conduits au cours des années 1990 dans le sud-ouest de la France ont permis de caractériser l'effet du couvert végétal sur le développement de l'épidémie de phomopsis du tournesol, un effet négatif sur le développement de la maladie étant observé en situation de couvert végétal réduit. Cette réduction du couvert pouvant être obtenue en jouant sur la densité de plante par $\mathrm{m}^{2}$ et l'azote ou « subie » naturellement dans les situations de haut de coteaux moins favorables à la croissance végétative du tournesol. Cet effet est très net sur le développement de Diaporthe helianthi, lorsque les conditions de pluviométrie sont moyennement favorables à l'épidémie. Il a été en revanche observé une absence d'effet les années où le climat est très favorable à l'épidémie, le poids des facteurs agronomiques devenant négligeable. Une inversion du phénomène a même été observée en 1992 lors d'une année marquée par une pluviométrie exceptionnelle en juin, rendant l'hygrométrie à l'intérieur de la culture facteur non limitant. Dans ce contexte climatique, les parcelles peu denses ou les situations de coteaux ont été plus affectées, a priori en raison d'une meilleure pénétration des ascospores dans la végétation. Des observations comparables sur l'incidence de la densité de végétation sont également observées en cultures de céréales notamment pour l'oïdium, maladie particulièrement sensible à ce facteur. Une forte disponibilité en azote à l'automne favorise le développement du phoma sur le colza [8].

\section{Autres méthodes : biofumigation, allélopathie, irrigation}

Parmi les autres méthodes, l'allélopathie est citée pour mémoire dans la mesure où des recommandations pratiques ne sont pas généralisées dans les systèmes de cultures européens. II s'agit de jouer sur l'ensemble des interactions biochimiques directes ou indirectes, positives ou négatives, d'une plante sur un autre organisme vivant (micro-organismes inclus) au moyen de métabolites secondaires, notamment les acides phénoliques, les flavonoïdes, les terpénoïdes et les alcaloïdes.
L'incorporation de ces substances allélopathiques dans la gestion de l'agriculture peut donc en théorie réduire l'utilisation d'herbicides, de fongicides et d'insecticides; et potentiellement diminuer l'impact des pesticides sur l'environnement aux conditions :

1) que la solution développée soit intrinsèquement suffisamment efficace seule ou associée à d'autres moyens de lutte dont celle faisant appel aux pesticides ;

2) que les effets négatifs ne l'emportent pas sur les effets positifs, certaines substances naturelles étant au moins aussi dangereuses que les substances issues de la chimie et de facto aujourd'hui peu évaluées.

S'agissant d'allélopathie, il est souvent fait référence à l'impact sur la croissance des adventices d'une culture.

La biofumigation fait l'objet d'une utilisation réduite limitée à la lutte contre les nématodes. Elle consiste à cultiver puis à incorporer des plantes notamment de la famille des crucifères dans le sol. La décomposition naturelle de cette biomasse libère des substances toxiques soufrées identiques à celles des fumigants interdits (isothiocyanates ou ITC) qui peuvent détruire certains pathogènes et ravageurs du sol dont les nématodes, c'est de fait le seul exemple d'utilisation de la méthode dans les conditions françaises notamment avant l'implantation d'une culture de betterave.

Une action sur des champignons du sol tels les verticilium, pythium et rhizoctone est revendiquée pour la méthode.

L'efficacité de la biofumigation doit être évaluée pour chaque maladie et ravageur. Les conséquences environnementales de la pratique et de sa généralisation via la libération des composés ITC dans l'atmosphère devront cependant faire l'objet d'une évaluation parallèle avant l'adoption généralisée et sans restriction de la technique.

L'effet de l'irrigation sur la dynamique épidémique des maladies des feuilles est mal cerné ou n'a qu'un impact limité en raison d'une humectation des feuilles en général plus courte que dans le cas d'une pluie. Pour obtenir un effet sur le développement des maladies, des systèmes complexes, faisant appel à des brumisateurs, sont nécessaires pour augmenter la pression de maladies lorsque la pluviométrie est insuffisantes. L'irrigation paraît, sauf exception, peu déterminante car les périodes d'irrigation sont des périodes de climat limitant sur le plan de l'humectation des feuilles (expérimentations conduite par I'ITCF au Magneraud à la fin des années 1980). II convient de pondérer ces résultats et ces observations par le fait que les zones concernées par l'expérimentation sont des zones caractérisées par des vents d'ouest réguliers qui sèchent rapidement le feuillage et ramènent l'hygrométrie de l'air à un niveau équiva- 
lent à une zone non irriguée dans un délai inférieur à 2 heures. Ces caractéristiques sont valables pour la plupart des régions françaises en fin de printemps et durant l'été.

A contrario le maintien d'eau à la surface du sol en phase de levée des plantes favorise des champignons telluriques tels le charbon des inflorescences du maïs, Sphacelotheca reiliana ou le mildiou du tournesol dû à Plasmopara halstdii. Le drainage naturel ou artificiel du sol limite les dégâts de ces organismes.

Une maladie telle que la hernie du chou due à Plasmodiophora brassicae sur colza est efficacement combattue par le chaulage et la remontée du pH du sol au-dessus de 6. Cette maladie est liée à des sols plus ou moins hydromorphes et acides.

Sur les insectes, le recours à l'irrigation est devenu systématique pour limiter les dégâts de pyrale du haricot sur soja Etiella zinckenella.

Un effet positif de la méthode est observé sur les acariens Tetranychus urticae voire les punaises Nezara viridula, arthropodes contrariés dans leur activité par l'irrigation.

Les acariens observés en grandes cultures, essentiellement Tetranychus urticae et les cicadelles dont celles observées sur maïs et sur blé, sont en général sensibles à la pluie comme à l'irrigation qui réduit leur population et perturbe leur activité.

II ne sera pas traité spécifiquement de la protection et du renforcement des organismes utiles importants via l'introduction de plantes appropriées en bordure de parcelle ou la valorisation $d$ 'infrastructures écologiques généralement des sites de production, souvent des zones tampons à vocation multiple, cette thématique limitée à la gestion des ravageurs nécessitant un développement spécifique.

\section{Conclusion}

Nous relèverons conformément aux listes de la directive sur l'utilisation durable des pesticides, l'existence d'une panoplie de moyens permettant d'éviter ou de compléter l'utilisation de produits phytopharmaceutiques mais aussi les nombreux paradoxes qui accompagnent leur mise en œuvre.

La gestion d'objectifs tout aussi nécessaires que sont la lutte contre l'érosion des sols et la gestion phytosanitaire des résidus de cultures fait apparaître les antagonismes les plus notables. Ces antagonismes empêchent, entre autres, une logique d'accumulation des techniques alternatives et militent pour qu'une hiérarchie dans les risques à gérer comme une bonne connaissance des bénéfices attendus des méthodes soit disponible en tenant compte des conditions de mise en œuvre. En complément de I'amélioration génétique, si la rotation, notamment celle qui fait alterner graminées et dicotylédones, reste la mesure de gestion la plus opérationnelle, sans impact sur la productivité de la culture, l'ensemble des autres méthodes présente toujours des termes d'ambivalence qu'il s'agit d'intégrer pour en tirer le meilleur parti. Les oléagineux outils facilitant la rotation constituent en cela une richesse à souligner pour une gestion durable de la lutte contre les bioagresseurs des cultures.

\section{RÉFÉRENCES}

1. Debaeke P, Orlando D. Simplification du travail du sol et évolution de la flore adventice : conséquences pour le désherbage à l'échelle de la rotation. In : Monnier G, Thévenet $G$, Lesaffre $B$, eds. Simplification du travail du sol. Paris (France) : INRA éditions, 1994 : 35-62.
2. Jouy L, Munier-Jolain N. Gestion de I'interculture, désherbage et protection des cultures. In : Du labour au semis direct : enjeux agronomiques, Conférence-débat INRA-ITCF. Salon International du Machinisme Agricole, février 2001, p 18-20, p. 22

3. House G], Parmelee RW. Comparison of soil arthropods and earthworms from conventional and no-tillage agroecosystems. Soil Tillage Res $1985 ; 5: 351-60$.

4. Haynes RJ. Swift Rs, Stephen RC. Influence of mixed cropping rotations (pasture-arable) on organic matter content, stable aggregation and clod porosity in a group of soils. Soil and Tillage Research $1991 ; 19$ : 77-87.

5. Beare MH, Hendrix PF, Coleman DC. Waterstable aggregates and organic matter fractions in conventional and no-tillage soils. Soil Sci Soc Am / 1994 ; 58 : 777-86.

6. Haynes RJ. Labile organic matter as an indicator of organic matter quality in arable and pastoral soils in New Zealand. Soil Biol Biochem 2000 ; 32 : 211-9.

7. Delos M, Eychenne N, Birba I, Fabry C. Étude des facteurs expliquant les fluctuations des attaques de Plasmopara halstedii en France. In: Proceedings of the 15th International Sunflower Conference, Toulouse, France, 12-15 June 2000. Vol. II, pp. I.43-I.48.

8. Aubertot JN, Dupeuple F, Grippon S, Penaud A, Pinochet X, Taverne M, Reau R. Phoma du colza : vers une protection intégrée. In: Les Rencontres Annuelles du CETIOM, 2002, 03-04 décembre. Paris, France : 22-30.

9. Chin MS, Kim SH, Park WM. Effect of planting date, plant density, nitrogen level, harvest date and benomyl treatment on Diaporthe phaseolorum var. sojae infection and germination of soybean seeds. J Agr Sc 1993 ; 35 : 99-108.

10. Tourvieille de Labrouhe $D$, Mestries E, Walser P. Quelles perspectives pour la lutte génétique visa-vis du mildiou du tournesol ? OCL $2005 ; 12$ : 85-93. 